\title{
SUPER-ELEMENT GLOBAL MODAL PARAMETERIZATION FOR EFFICIENT INCLUSION OF HIGHLY NONLINEAR COMPONENTS IN MULTIBODY SIMULATION
}

\author{
F. NAETS \\ W. DESMET \\ Department of Mechanical Engineering, Celestijnenlaan 300B, B-3001 Heverlee (Leuven), Belgium
}

\begin{abstract}
Over the last decades, higher and higher accuracy is expected from flexible multibody models. Accurate flexibility descriptions and detailed contact algorithms have been mostly developed with this aim. In practice, linearized models are often used at the expense of severe accuracy loss in order to improve simulation times. Several multibody packages have also been developed for the simulation of nonlinear components, but these typically lead to elevated simulation times. Alternatively, a cosimulation with a nonlinear finite element package can be performed, but this leads to very inefficient schemes. In order to allow a generic introduction of nonlinear components in multibody simulation packages, this paper introduces a novel nonlinear model reduction technique for nonlinear components, namely the Super-element Global Modal Parameterization (SEGMP). The approach is aimed at reducing complex models of nonlinear components, while still taking the important nonlinear effects into account, in order to create a relatively simple model which can easily be coupled with other components in a multibody model. The proposed approach is based on the GMP formulation which is a nonlinear system level model reduction technique for (flexible) multibody systems. This work discusses the reduction procedure and different choices for the reduction space. Moreover, a strong focus is put on an appropriate choice of coordinates in order to allow for a convenient interfacing with multibody descriptions. Finally, the approach is demonstrated by the simulation of a slider-crank mechanism with a nonlinearly deforming crank.
\end{abstract}

\section{INTRODUCTION}

Over the years, multibody models have become increasingly popular and the complexity has risen accordingly [1]. Current industrial multibody models contain a high number of bodies of which many are modeled as flexible. Also the accuracy expected from these models has risen considerably over the last years. In many applications, nonlinear components, such as bushings, bearings or components undergoing large deformations (e.g. twist-beam suspensions) are present. In order to accurately capture the behavior of the system, the inclusion of nonlinear effects is essential. In practice there are two techniques in use to include nonlinear components:

- use approximate lumped force-elements [2];

- use fully nonlinear finite element model (possibly in co-simulation) [3].

Due to the high computational cost typically associated with the inclusion of a nonlinear finite element model in a simulation, the most popular approach is to use lumped force-elements. The approach proposed by Ambrosio [2] allows capturing the global nonlinear behavior of bushing-type elements in an efficient fashion. The approach however does not allow for the inclusion of the internal dynamic behavior of the nonlinear component. In practice nonlinear components, such as bushings, are often even replaced by a linearized $6 \times 6$ stiffness matrix in commercial software. This is common practice for the inclusion of bearings or for the modeling of anti-roll bars in vehicles. However, these components don't behave like 
linear springs in reality and moreover these approaches also do not allow the inclusion of the internal dynamics of the component.

In order to meet the drawback of having to choose between the two extremes of large computational load or low accuracy, the authors propose a novel reduction technique for nonlinear components, namely Super-Element Global Modal Parameterization (SE-GMP). This technique allows the reduction of a complex nonlinear FE model into a compact dynamical model which can be included in a MBD-model. This approach is a variation on the Sub-System GMP method, which can be viewed as a generalization of the super-element approach proposed by Agrawal et al. [4], for the inclusion of sub-mechanisms. Both these approaches are based on the Global Modal Parameterization, which was first introduced by Brüls [5]. The original formalism used a dynamic parameterization to describe the rigid motion of the system and a linearized flexible deformation, leading to a considerable reduction in the amount of degrees-of-freedom (DOFs). Sub-System Global Modal Parameterization has been previously introduced as a technique for system-level reduction of sub-mechanisms of flexible multibody systems [6,7]. In this approach, a minimal set of rigid degrees-of-freedom (DOFs) is used to dynamically parameterize the configuration of a sub-system which is part of a larger system. The formulation also allows the sub-mechanism to move as a whole, in contrast with the regular GMP method in which the system should be defined with respect to an absolute reference frame.

In this work, the formalism is extended to include general degrees-of-freedom, which do not have to be rigid motion DOFs, for the nonlinear motion parameterization. By, for example, using large deformation patterns to parameterize the motion of a component, nonlinear stiffening phenomena can be simulated accurately. Moreover, in order to facilitate the use of a SE-GMP reduced component in a general multibody model, a new set of generalized DOFs is proposed for the reduced model. Previously, the configuration of the floating reference frame, the parameterization DOFs and flexible deformation participation factors served as reduced DOFs [6]. Unfortunately, these DOFs lead to a very complex description of the connections with other components. Another benefit of the SE-GMP approach is the fact that it is formulated such that the interface DOFs of the reduced component are used for the description of the configuration of the SE-GMP model, augmented with flexible deformation participation factors. The aim of this approach is to obtain a general procedure for the reduction of nonlinear components which leads to efficient models which can be easily inserted in existing multibody codes.

This work first discusses the use of the GMP reduction formalism for the reduction of general nonlinear systems. Next, the choice of coordinates and equations of motion to reach the Super-Element description are discussed. Finally, in order to demonstrate the proposed approach, a numerical simulation of a highly flexible slider-crank mechanism is considered. During the motion of this system, the crank exhibits nonlinear stiffening effects. A comparison is made between a full nonlinear model, a floating-frame-of-reference component-mode-synthesis (FFR-CMS) model and a model in which the crank is reduced with the SE-GMP approach. The model with the SE-GMP component shows good accuracy whereas the FFR-CMS model diverges strongly from the original model. 


\section{Super-Element Global Modal Parameterization}

This work aims at the reduction of nonlinear mechanical components for which the Lagrangian, as function of the generalized coordinates $q$, can be written as ${ }^{1}$ :

$$
\mathscr{L}=\frac{1}{2} \dot{q}^{T} M^{q q}(q) \dot{q}-\mathscr{V}(q)+\mathscr{W}
$$

with

- $q$ is a vector of $n^{q}$ generalized coordinates, which define the configuration of the system.

- $M^{q q}(q)$ is the configuration-dependent mass matrix.

- $\mathscr{V}(q)$ is the nonlinear internal potential energy. Only potential energy due to structural deformation is considered in this term.

- $\mathscr{W}$ denotes the work due to external loads.

In this equation, the nonlinear behavior is only dependent on the configuration $q$ and not on the velocities or accelerations. This means that this work does not consider velocity dependent nonlinear effects such as frequency-dependent damping, but in general there is no theoretical objection against the inclusion of this kind of effects. Moreover, also internal energy dissipation is omitted in this work. In the case where the undamped modes are still sufficiently accurate for the damped system, the inclusion of damping is however straightforward.

The SE-GMP methodology for the efficient inclusion of nonlinear components in multibody models consists of two important aspects:

- reduction of a general nonlinear component through the GMP approach,

- creating a super-element formulation by using global coordinates for the interface nodes.

This chapter is also split in these two tracks. First the reduction of a nonlinear mechanical component by a GMP formalism is presented. The GMP reduction is described by using a variable projection and a constant projection space, which is a novel approach in this framework. This GMP reduction leads to an efficient formalism to evaluate the nonlinear and dynamical behavior of the component. In the second subsection, the use of a parameterization with flexible DOFs and a specific choice of coordinates (super-element coordinates) is discussed which allows for an easy interfacing with other components. The equations of motion using these coordinates are presented. The easy interfacing is essential to allow a practical coupling with existing multibody packages.

2.1. GMP for nonlinear components. In the Global Modal Parameterization, a dynamical parameterization of a system is performed, where a minimal set of dynamically varying parameters are introduced to describe the nonlinear motion of a system and a linearized deviation from this motion is added as well $[5,8]$.

In order to perform this nonlinear parameterization, the model is computed for a grid of $m$ possible configurations, and the dynamical behavior of these configurations is reduced and then stored during the pre-processing. During the actual simulation, the stored data of the reduced model is used to evaluate the model. For the reduction of nonlinear components, this framework is still the same as in a regular GMP reduction of a mechanism.

\footnotetext{
${ }^{1}$ Matrices are denoted with a uppercase letters and vector by lowercase letters. Superscripts denote to which kind of DOFs the matrix/vector relates. E.g. $M^{q q}$ is a matrix which gives a property expressed in $q$-coordinates and has size $n^{q} \times n^{q}$.
} 
The basis of the GMP-reduction scheme is a coordinate transformation onto a nonlinear manifold [5], in which the generalized unreduced coordinates $q$ are given as a function of the reduced coordinates $\theta$ and $\delta$ :

$$
q=\rho(\theta)+\Psi^{q \delta}(\theta) \delta
$$

In this formula, $\theta$ is a generalized DOF describing the nonlinear motion of the system and $\delta$ are the DOFs which only have a linearized effect. A nonlinear function $\rho$ describes the unreduced coordinates as a function of $\theta$. To this, a linearized deviation is added. These are further referred to as the deviation DOFs because they describe a deviation from the main motion. In the general GMP formulation, the linearized projection $\Psi^{q \delta}$ is also function of $\theta$ due to changing dynamics of the component. The GMP DOFs are grouped together in the vector $\eta$ :

$$
\eta=\left[\begin{array}{l}
\theta \\
\delta
\end{array}\right]
$$

In a GMP reduction for a mechanism [5], the global parameter $\theta$ is used to describe the rigid motion of the system. For a general nonlinear component however, this definition has to be generalized. In this context, $\theta$ can be associated with any degree-of-freedom which has an important effect on the nonlinear system dynamics. As in the regular GMP case it has to be mentioned that the storage requirements and computational load rises exponentially with the number of nonlinear configuration parameters $\theta$. Therefore this approach is mainly suitable for applications in which only a limited number of nonlinear phenomena are important. Alternatively in some applications it might also be possible to split a component up into multiple SE-GMP models such that the load rises only linearly. In practice, this will often lead to the linking of $\theta$ to a degree-of-freedom which is expected to have large displacements and/or deformations. For a cantilever beam for example, this can be the lateral displacement of the free tip, as will be shown in Sec. 3 . In all cases, $\delta$ acts as the modal amplitudes of the linearized deviations of the system, as shown in Fig. 1. With

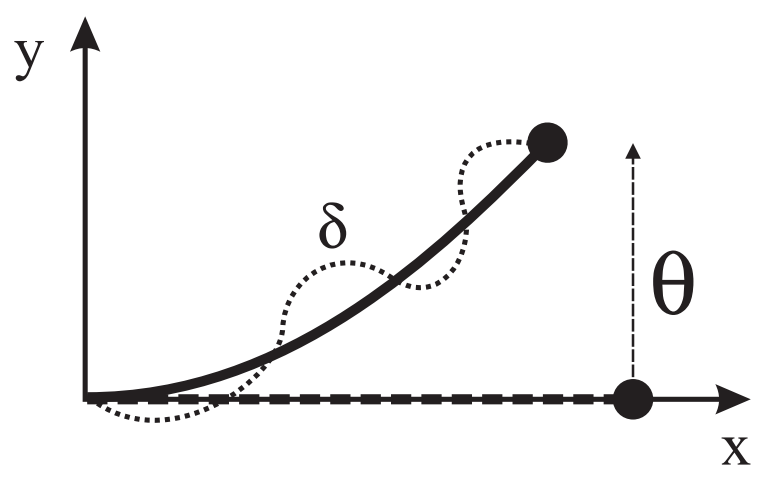

FIGURE 1. GMP reduced coordinates.

this definition for the degrees of freedom, the velocity projection, which is important for the derivation of inertial forces, can be written as [5]:

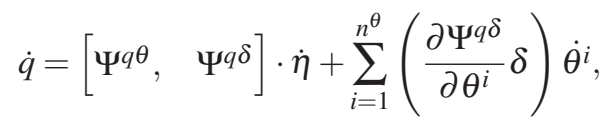

with

$$
\Psi^{q \theta}=\frac{\partial \rho}{\partial \theta}
$$


In this equation, all projection vectors $\Psi^{q}$ are dependent on $n^{\theta}$ dynamic parameters $\theta$, but this dependency is not shown explicitly in the equation for the sake of brevity. It is important to notice that due to this dependency, the velocity projection also contains terms dependent of the linearized deformation $\delta$.

To perform the reduction, the system is evaluated over a set of possible configurations for $\theta$. In order to have a reference, the component has to be fixed to prevent rigid body motion. This fixation will also serve as a reference frame when considering the super-element description in Sec. 2.2. Many possiblities exist to perform this fixation and in this work a tangent frame approach is adopted. In this approach, one of the DOFs of the system is held fixed. The tangent frame approach is chosen for its ease of implementation, but all theory presented holds just as well for a Buckens frame approach or others. With a set of $\theta$ 's defined with respect to this reference-frame, the basis configurations of the system $\rho$ for each of these $\theta$ 's is defined by minimizing the internal potential energy $\mathscr{V}$ :

$$
\begin{array}{ll}
\underset{\rho \in \mathbb{R}}{\operatorname{minimize}} & \mathscr{V}(\rho) \\
\text { subject to } & \rho_{\theta}=\theta .
\end{array}
$$

This is a minimization problem subject to constraints to define the configuration. The constraints $\rho_{\theta}=\theta$ state that the configuration vector has to equal the desired $\theta$ 's at their respective positions. In general this problem will have to be solved numerically. By starting the loop over the different configurations from the undeformed state, the previously obtained configurations can be used as starting values for the optimization, which will typically lead to faster convergence.

Once the configuration for a given $\theta$ is known, the model reduction can be performed by projecting the dynamics on the appropriate projection space. The choice of this projection space is discussed in Sec. 2.1.1. With the projection space known, the reduced equations of motion can be derived, as discussed in Sec. 2.1.2

2.1.1. Selection of reduction space. One of the most important choices for the dynamic behavior of the reduced model, is the choice of the linearized projection space $\Psi^{q \delta}$. In order to get a proper dynamic behavior of the reduced system, this projection space should be carefully chosen.

In this work, the projection spaces are all based on eigenmodes, as in previous work on the GMP reduction method [5, 9]. In literature, other reduction techniques, such as Krylov and Gramian based projection, have been proposed for linear dimensional reduction [10,11]. These techniques could also be exploited in the framework of a GMP reduction, but are not further investigated here.

In the case of a single nonlinear component, as considered in this work, no constraint equations are present in the equations of motion for the unconnected component. This leads to a system of ordinary differential equations (ODEs) for the equations of motion, in contrast to GMP for mechanisms where a set of differential algebraic equations (DAEs) were obtained. If constraints are present in the equations of motion, a variable mode space is necessary because for every configuration, the modes have to comply with the constraints and this cannot be achieved with a constant projection space. In the context of component reduction without constraints however, it is also possible to exploit a constant projection space.

These two projection techniques have the following properties:

Variable reduction space: In this case the projection space is calculated and stored for all $m$ different combinations of $\theta$ for the component:

$$
\Psi_{t o t}^{q \delta}=\left[\Psi^{q \delta}(\theta(1)), \Psi^{q \delta}(\theta(2)), \ldots, \Psi^{q \delta}(\theta(m))\right] .
$$

The complete projection space over all configurations is obtained by concatenating all projection matrices for all $m$ configurations. During the actual simulation an interpolation has to be performed 
between the relevant spaces dependent on the configuration of the system in order to extract the required $\Psi^{q \eta}(\theta)$. By using a variable projection space, the space can be optimized to approximate the system dynamics at each configuration with a minimal set of modes. For systems with strongly variable dynamics, this leads to the largest reduction potential.

However, the variable reduction space can also lead to considerable difficulties, such as issues due to mode-crossing and -veering [12]. Due to the changing dynamics of the system, the modes and their eigenfrequencies change and at certain configurations, some modes will morph into each other. However, continuity of the modes has to be guaranteed due to Eq. (4). As will be discussed in this section, the inertial forces are dependent on the derivatives of the projection space and large derivatives can lead to numerical problems.

Constant reduction space: In case the component is described by a set of ODE's, it is also possible to define a constant projection space. In this case, a full variable projection space with the eigenmodes $\Psi_{t o t}^{q \delta}$ is first constructed. All these modes are then grouped together and a singular value decomposition (SVD) is performed on this matrix:

$$
\Psi_{\text {tot }}^{q \delta}=U \Sigma V^{T} .
$$

From this decomposition, the vectors in $U$ corresponding to the $n^{\delta 2}$ largest singular values in $\Sigma$ are kept:

$$
\Psi^{q \delta}=\left[\begin{array}{llll}
U_{1}, & U_{2}, & \ldots, & U_{n^{\delta}}
\end{array}\right] .
$$

This SVD-decomposition allows to select a minimal set of modes which span an optimal approximation of the set of variable eigenmodes. Other approaches to create this constant space exist in literature, but are not investigated here [13].

This approach has as major advantage that the modal spaces $\Psi^{q \delta}\left(\theta^{i}\right)$ on which the SVDcomposition is based, do not need to be continuous in $\theta$ and that all inertial forces associated with the derivatives of $\Psi^{q \delta}$ disappear (see Sec. 2.1.2). Unfortunately this method compromises the reduction of the amount of DOFs, because a larger set of constant modes is required to span a similar space as the variable modes. In general, a larger number of deviation DOFs $\delta$ will be required for a GMP model with a constant reduction space in order to get similar accuracy as the model with variable reduction space. In the construction of this projection space, two choices have to be made: the number of modes to construct the eigenmode space and the number of modes to be kept for the resultant constant space. The number of modes for the constant space can easily be tuned based on the decay of the singular values. It is however important to pay attention to the number of modes for the eigenmode-space because high-frequency modes can easily distort the SVD-space if no appropriate weighting is applied, but this aspect is not further investigated here [14].

Both of these approaches are theoretically valid and the choice for one or the other approach depends on a trade-off between:

- dimensionality reduction,

- accuracy,

- (numerical) stability.

The limitation on dimensional reduction and accuracy for the constant reduction space can be seen by considering the example of a cantilever beam with large flexible deformation (see Sec. 3). In Fig. 2 the first

\footnotetext{
$2 n^{\delta}$ for the constant reduction can be different from the number of modes used to construct the total projection space on which the constant space is based.
} 

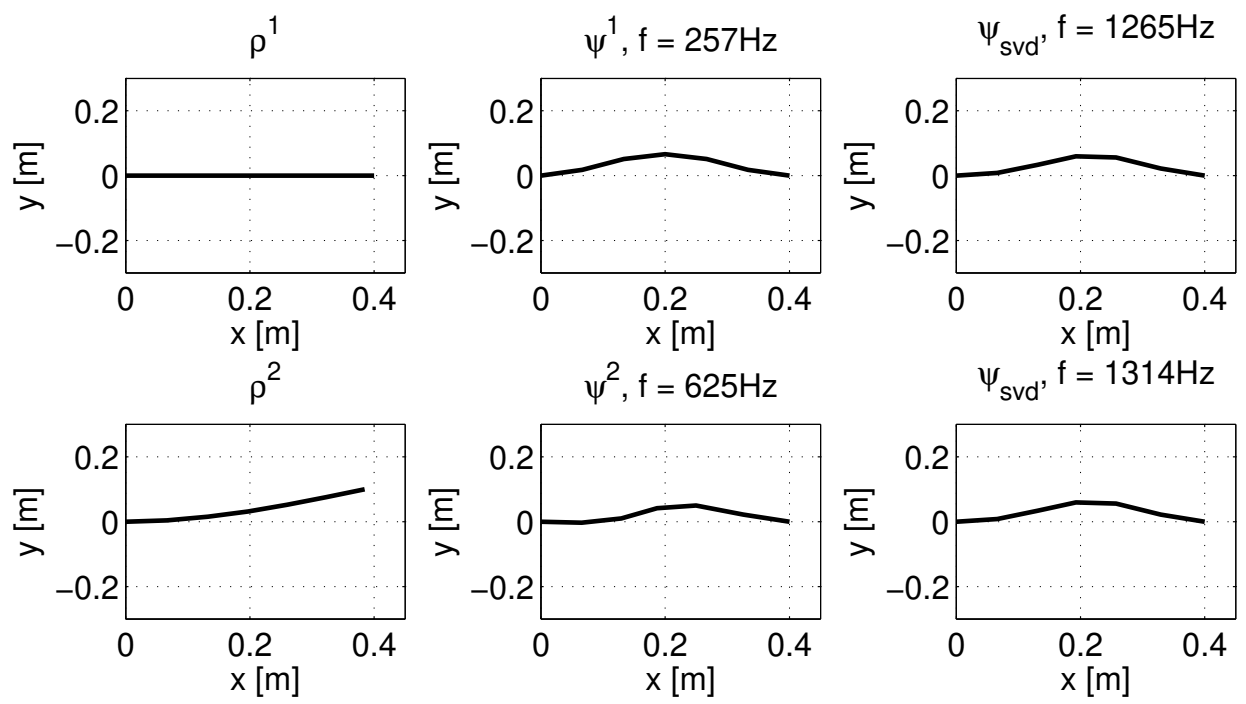

FIGURE 2. Variable vs. constant reduction space: reference configuration, variable mode, constant mode.

fixed-fixed eigenmode of the beam is shown for 2 configurations of a cantilever beam and also the resultant first SVD-mode is shown. Even though the SVD-pattern is similar to both eigenmodes, it will require more energy to apply this deformation and the eigenfrequency will be shifted upwards in both configurations. This leads to a higher error for the model with the constant projection space for the same number of modes. For many systems, which only show small differences in the space spanned by the modes, this effect will be minimal but for extreme cases this could be an important effect. In order to obtain the same frequency content with a constant projection space, a higher number of modes will be required than in the case of a variable projection space.

2.1.2. GMP equations of motion. The equations of motion are derived from the Lagrangian of the system under consideration, as given in Eq. (1), by applying Hamilton's principle [5]:

$$
\frac{d}{d t}\left(\frac{\partial \mathscr{L}}{\partial \dot{\eta}}\right)-\frac{\partial \mathscr{L}}{\partial \eta}=0
$$

In order to derive the equations for the reduced DOFs $\eta$, the coordinate-transformations from Eq. (2) and (4) have to be introduced. This leads to equations of motion of the form:

$$
g_{\text {iner }}^{\eta}(\ddot{\eta}, \dot{\eta}, \eta)+g_{\text {int }}^{\eta}(\eta)=g_{\text {ext }}^{\eta} .
$$

In this equation $g_{\text {iner }}^{\eta}$ are the generalized inertial loads onto the reduced coordinates and expressed as a function of the reduced positions, velocities and accelerations. $g_{\text {int }}^{\eta}$ are the internal elastic forces and $g_{\text {ext }}^{\eta}$ are the external loads for the system described by the reduced coordinates. These forces are derived from the linearizations around the nonlinear motion $\rho(\theta)$ analogous as for the equations of motion for a GMP model for mechanisms [5, 9]:

: Inertial forces The inertial forces are derived from the kinetic energy. In the assumption of $n^{\delta}$ sufficiently small deviations $\delta$ from the main motion, the unreduced mass matrix is only dependent 
on $\theta$ and the kinetic energy can be written as:

$$
\begin{gathered}
\mathscr{K}=\frac{1}{2} \dot{\eta}^{T}\left[\Psi^{q \theta}+\frac{\partial \Psi^{q \delta} \delta}{\partial \theta}, \Psi^{q \delta}\right]^{T} M^{q q}\left[\Psi^{q \theta}+\frac{\partial \Psi^{q \delta} \delta}{\partial \theta}, \Psi^{q \delta}\right] \dot{\eta} \\
=\frac{1}{2} \dot{\eta}^{T}\left(M_{1}^{\eta \eta}+\sum_{i=1}^{n^{\delta}} M_{2 i}^{\eta \eta} \delta^{i}+\sum_{i=1}^{n^{\delta}} \sum_{j=1}^{n^{\delta}} M_{3 i j}^{\eta \eta} \delta^{i} \delta^{j}\right) \dot{\eta} \\
=\frac{1}{2} \dot{\eta}^{T} M^{\eta \eta} \dot{\eta}
\end{gathered}
$$

The mass matrices in Eq. (12) are defined as:

$$
\begin{aligned}
M_{1}^{\eta \eta}= & \left(\Psi^{q \eta}\right)^{T} M^{q q}\left(\Psi^{q \eta}\right) \\
M_{2 i}^{\eta \eta}= & \left(\Psi^{q \eta}\right)^{T} M^{q q}\left[\frac{\partial \Psi^{q \delta^{i}}}{\partial \theta}, 0\right]+\left[\frac{\partial \Psi^{q \delta^{i}}}{\partial \theta}, 0\right]^{T} M^{q q} \Psi^{q \eta} \\
M_{3 i j}^{\eta \eta}= & {\left[\frac{\partial \Psi^{q \delta^{i}}}{\partial \theta}, 0\right]^{T} M^{q q}\left[\frac{\partial \Psi^{q \delta^{j}}}{\partial \theta}, 0\right] } \\
& +\left[\frac{\partial \Psi^{q \delta^{j}}}{\partial \theta}, 0\right]^{T} M^{q q}\left[\frac{\partial \Psi^{q \delta^{i}}}{\partial \theta}, 0\right]
\end{aligned}
$$

Applying Hamiltons principle (9), the inertial forces are derived analogous as described by Brüls [5] and Naets [9].

$$
g_{\text {iner }}^{\eta}=M^{\eta \eta} \ddot{\eta}+\left(\sum_{l=1}^{n^{\eta}} \frac{\partial M^{\eta \eta}}{\partial \eta^{l}} \dot{\eta}^{l} \dot{\eta}-\frac{1}{2} \frac{\partial \dot{\eta}^{T} M^{\eta \eta} \dot{\eta}}{\partial \eta}\right)
$$

These inertial forces consist of the regular acceleration dependent inertial forces and velocity dependent generalized gyroscopic forces. It is easy to see, that in order to determine the derivatives of the mass-matrices in Eq. (14)-(16), many derivatives of the projection space are necessary and even second-order derivatives are required. These derivatives are typically computed by finite differentiation, even though more robust automatic schemes exist in literature for sensitivity analysis [15], which easily leads to numerical problems, especially in the case of mode-veering and -crossing. Moreover, these derivatives also make the evaluation of the gyroscopic inertial forces quite involved.

As was stated in Sec. 2.1.1, it is also possible to use a constant reduction space for the nonlinear component reduction. In this case, the deviation modes $\Psi^{q \delta}$ are constant and no derivatives are present:

$$
M_{2}^{\eta \eta}=M_{3}^{\eta \eta}=0
$$

which greatly simplifies the computation of the inertial forces, leading to reduced computational load, and allows a more robust formulation.

: Internal forces The internal forces for the reduced model are derived from a Taylor-expansion of the internal potential energy $\mathscr{V}$ :

$$
\begin{aligned}
\mathscr{V}\left(\rho(\theta)+\Psi^{q \delta} \delta\right)=\mathscr{V}(\rho(\theta))+\left(\Psi^{q \delta} \delta\right)^{T} \frac{\partial \mathscr{V}}{\partial q} & +\frac{1}{2} \delta^{T}\left(\Psi^{q \delta}\right)^{T} \frac{\partial^{2} \mathscr{V}}{\partial q^{2}} \Psi^{q \delta} \delta+O\left(\delta^{3}\right)
\end{aligned}
$$


In this equation, the nonlinear function and derivatives are evaluated at $q=\rho(\theta)$. The third order terms in $\delta$ will lead to second-order terms in the forces and are assumed negligible due to small $\delta$. The internal forces are obtained by applying Hamilton's principle to this internal potential energy equation:

$$
\begin{aligned}
g_{\text {int }}^{\theta}= & \left(\Psi^{q \theta}\right)^{T} \frac{\partial \mathscr{V}}{\partial q}+\left(\left(\Psi^{q \theta}\right)^{T} \frac{\partial^{2} \mathscr{V}}{\partial q^{2}} \Psi^{q \delta} \delta+\frac{\partial \mathscr{V}}{\partial q} \frac{\partial \Psi^{q \delta} \delta}{\partial \theta}\right) \\
& +O\left(\delta^{2}\right), \\
= & \left(\Psi^{q \theta}\right)^{T} \frac{\partial \mathscr{V}}{\partial q}+\left(\Psi^{q \theta}\right)^{T} \frac{\partial^{2} \mathscr{V}}{\partial q^{2}} \Psi^{q \delta} \delta, \\
g_{i n t}^{\delta}= & 0+\left(\Psi^{q \delta}\right)^{T} \frac{\partial \mathscr{V}}{\partial q}+\left(\Psi^{q \delta}\right)^{T} \frac{\partial^{2} \mathscr{V}}{\partial q^{2}} \Psi^{q \delta} \delta, \\
= & \left(\Psi^{q \delta}\right)^{T} \frac{\partial^{2} \mathscr{V}}{\partial q^{2}} \Psi^{q \delta} \delta .
\end{aligned}
$$

The second-order effects with respect to $\delta$ remaining in Eq. (20) can also be neglected in the assumption of sufficiently small $\delta$. Since the internal energy is minimized for the deviation, also the term dependent on the derivative of the projection space in Eq. (20) is zero and can be removed from the equations. In contrast to the GMP reduction for mechanisms [5], the first derivative of the potential energy will not disappear in these equations because the system is not in an absolute minimum of the internal energy for a given $\rho(\theta)$. The first derivative (2nd term) will however disappear in Eq. (23) because $\rho(\theta)$ is determined by minimizing the internal energy for $\delta$. It is important to notice that an internal force $g_{\text {int }, 0}^{\theta}$ now also exists when no deviation $\delta$ is present. Considering that the first derivatives of the internal energy are the internal forces for a given configuration $\rho(\theta)$, this leads to:

$$
g_{i n t, 0}^{\eta}=\left[\begin{array}{c}
\left(\Psi^{q \theta}\right)^{T} g_{i n t}^{q}(\rho(\theta)) \\
0_{n^{\delta} \times 1}
\end{array}\right] .
$$

A reduced stiffness matrix $K^{\eta \eta}$ can be defined to take the dependency of the internal forces on $\delta$ into account. Because the second derivative of the internal energy with respect to $q$ is simply the unreduced tangent stiffness matrix $K^{q q}$ in the configuration $\rho(\theta)$ :

$$
K^{\eta \eta}=\left[\begin{array}{ll}
0_{n^{\theta} \times n^{\theta}} & \left(\Psi^{q \theta}\right)^{T} K^{q q} \Psi^{q \delta} \\
0_{n^{\delta} \times n^{\theta}} & \left(\Psi^{q \delta}\right)^{T} K^{q q} \Psi^{q \delta}
\end{array}\right] .
$$

The internal forces can then be summarized as:

$$
g_{i n t}^{\eta}=g_{\text {int }, 0}^{\eta}+K^{\eta \eta} \eta
$$

It is important to notice that in this equation $K^{\eta \eta}$ is not the tangent stiffness matrix of the internal forces of the reduced model. The actual tangent stiffness matrix will also have non-zero elements in the first columns. 
: External forces The reduced external forces $g_{\text {ext }}^{\eta}$ can be determined very straightforwardly by projection from the unreduced forces $g_{\text {ext }}^{q}$ as in the GMP reduction for mechanisms:

$$
g_{\text {ext }}^{\eta}=\left[\Psi^{q \theta}+\frac{\partial \Psi^{q \delta} \delta}{\partial \theta}, \quad \Psi^{q \delta}\right]^{T} g_{\text {ext }}^{q} .
$$

Here again, the choice for a constant projection space can significantly simplify the projection of the external forces and limit the storage requirements for the reduced model.

The above described reduced forces can be combined to analyze the behavior of a single nonlinear component. In general, a considerable efficiency gain is possible due to the possibility of a strong reduction in the number of DOFs through the projection on the main system dynamics. This kind of model will also allow faster simulation because the number of DOFs which amount to the nonlinear behavior of the system is reduced with respect to e.g. a nonlinear FE simulation.

2.1.3. Interpolation for the reduced model. Another important aspect of the GMP reduced model is the interpolation used for the evaluation of the model in a certain configuration of $\theta$. Many formalisms have been introduced in literature for the interpolation of models, mainly in the context of model reduction for parameterized systems $[16,17]$. However, due to the large number of interpolations, one for each iteration/timestep, in the evaluation of the GMP model a simple linear interpolation is chosen in this work. This choice might require a finer discretization of the interpolation grid but does lead to a very efficient online interpolation. Further research should however be performed to exploit more advanced model interpolation schemes for GMP models.

2.2. Super-Element GMP description. The previously described reduction scheme leads to an efficient description of a fixed component or system. However, in order to use this reduction strategy for subcomponents in systems, the description should be expressed with respect to a moving reference frame and provide an efficient interfacing with other components.

In order create a description with respect to a moving reference frame, the same methodology as outlined by the Sub-System GMP (SS-GMP) approach is employed [6]. In this methodology, the reduced system is described with respect to a reference frame which is attached to the reduced system. This reference frame attached to the component is referred to as the component reference frame (CRF).

In this description three different kinds of degrees-of-freedom exist to describe the configuration of the reduced system (Fig. 3a):

- rigid DOFs $q_{0}$ to describe the position and orientation of the reference frame attached to the component:

$$
q_{0}=\left[\begin{array}{l}
x_{0} \\
p_{0}
\end{array}\right],
$$

- the nonlinear degrees-of-freedom $\theta$ which determine the nonlinear configuration of the system with respect to the CRF,

- modal amplitudes $\delta$ to determine the linearized deviation from the global and nonlinear motion.

These coordinates can be easily linked to the GMP reduction for a single component, since the same coordinate definitions are still used with the only addition of the motion of the CRF. This leads to the Sub-System 


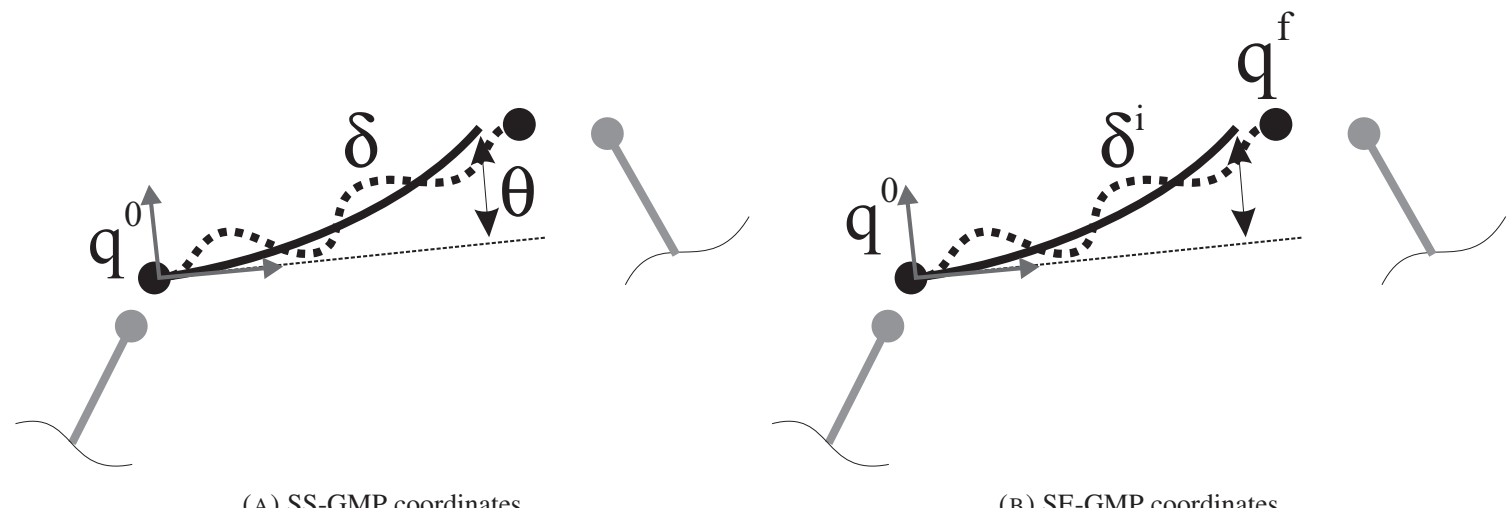

FIGURE 3. SS-GMP and SE-GMP coordinates (black: nonlinear component, grey: other components)

GMP coordinates $q_{s s}$ :

$$
q_{s s}=\left[\begin{array}{c}
q_{0} \\
\theta \\
\delta
\end{array}\right] .
$$

However, by using the SS-GMP description, it is fairly complicated to define connections with other components. The global positions of the interface coordinates are described by a nonlinear equation and the resulting constraint equations will be highly nonlinear. In general this will lead to a complex set of equations and including this scheme into an existing multibody package might prove overly complicated.

For interfacing with other components in a system simulation, a new set of generalized coordinates is introduced for the Super-Element GMP approach proposed in this work. In this description global coordinates are used for the interface DOFs, as shown in Fig. 3b. This leads to a new set of degrees-of-freedom for the SE-GMP model:

- rigid DOFs $q_{0}$ to describe the motion of the CRF in global coordinates,

- interface DOFs $q_{f}$ to describe the motion of the interface nodes in global coordinates,

- nonlinear DOFs $\theta$ to describe the nonlinear motion of the component,

- modal amplitudes $\delta$ to describe the linearized deviations.

These degrees-of-freedom however do require an appropriate coordinate projection to link them to the DOFs of a GMP reduced component. This coordinate projection is discussed in Sec. 2.2.1.

Finally these new coordinates also lead to a new set of equations of motion. The equations of motion employing these degrees-of-freedom are presented in Sec. 2.2.2.

2.2.1. Coordinate transformation. In order to allow a convenient description of the connection of a GMP reduced component with other components of a system, global coordinates are used to describe the interface DOFs of the reduced component. Because these global coordinates should be converted back to the original internal GMP DOFs, an appropriate choice of projection space has to be used. This problem is very similar to the one in super-element modeling of flexible bodies [18, 19].

A typical point of discussion is the definition of the reference frame attached to the component. As mentioned before, a tangent frame approach, where the reference frame is rigidly attached to one of the nodes of the system, is employed in this work. Moreover, an interface node is chosen to connect the frame, 
as shown in Fig 3b. Other choices are possible, but this allows a very straightforward transformation of one of the interface coordinates.

In order to allow a one-to-one transformation for the other interface DOFs, a set of Craig-Bampton modes [20] is used for the projection space. This leads to a set of fixed-fixed internal modes, where the interface DOFs are fixed, and static modes for the interface DOFs. This method has as main disadvantage that the reduction potential is strongly compromised in the case of many interface DOFs. With this choice of deviation modes, the modal amplitudes can be split up into two parts:

- $\delta_{f}$ for the modal amplitudes of the interface modes,

- $\delta_{i}$ for the modal amplitudes of the internal flexible modes.

The internal amplitudes $\delta_{i}$ are the modal amplitudes present in the final DOF-vector of the component. Due to the use of global coordinates for the interface nodes, the interface mode amplitudes $\delta_{f}$ are intermediate DOFs which have to be obtained from the global coordinates. Similar to the modal amplitudes, also the nonlinear DOFs $\theta$ can be divided into:

- $\theta_{f}$ for the nonlinear DOFs which are associated with interface nodes,

- $\theta_{i}$ for the internal nonlinear DOFs.

The use of $\delta_{f}$ and $\theta_{f}$ is necessary to evaluate the GMP reduced model of the component, but does not appear in the final coordinate vector $q_{s e}$ for a SE-GMP model:

$$
q_{s e}=\left[\begin{array}{c}
q_{0} \\
q_{f} \\
\theta_{i} \\
\delta_{i}
\end{array}\right] .
$$

In order to allow an appropriate use of the GMP reduced model, these coordinates have to be transformed internally in the model to the SS-GMP coordinates $q_{s s}$ from Eq. (29) [6]. The local GMP coordinates can then be split up:

$$
\theta=\left[\begin{array}{c}
\theta_{f} \\
\theta_{i}
\end{array}\right] \text { and } \delta=\left[\begin{array}{c}
\delta_{f} \\
\delta_{i}
\end{array}\right] .
$$

The back-transformation to the unreduced coordinates $q$ is also performed based on the SS-GMP coordinates:

$$
q=x_{0}+R_{0}\left(\rho(\theta)+\Psi^{q \delta} \delta\right),
$$

in which $R_{0}$ is a generalized rotation matrix function of the orientation $p_{0}$ of the CRF. As described by Naets [6], the unreduced velocities have to be obtained with respect to the CRF $\dot{q}^{\prime}$ in order to evaluate the inertial forces based on the reduced model in the CRF. These velocities can be obtained through projection [7]:

$$
\dot{q}^{\prime}=C\left(q_{s s}\right) \dot{q}_{s s}=N(\eta) D\left(q_{0}\right) \dot{q}_{s s} .
$$

In this equation $D$ is a projection matrix which takes the rotation of the CRF into account and $N$ is the projection matrix with the motion modes, a concatenation of the rigid body motion modes for the CRF and the projection space. However, for the use of SE-GMP coordinates, an additional transformation from $q_{s e}$ to $q_{s s}$ is necessary. No transformation is required for $q_{0}, \theta_{i}$ and $\delta_{i}$, but the interface coordinates $q_{s e, f}=$ $\left[\begin{array}{ll}\theta_{f}, & \delta_{f}\end{array}\right]^{T}$ have to be projected. In general $\theta_{f}$ and $\delta_{f}$ can be obtained by solving the nonlinear equation:

$$
\left(R_{0}\right)^{T}\left(q_{f}-q_{0}\right)-\left(\rho_{f}\left(\theta_{f}, \theta_{i}\right)+\Psi^{q_{f}} \delta_{f} \delta_{f}\right)=0 .
$$


However, solving this nonlinear equation on-line will prove computationally expensive. By an appropriate choice of parameterization, the equations for $\theta_{f}$ and $\delta_{f}$ can be decoupled. If $\theta_{f}$ is directly associated with one of the local DOFs, the solution for $\theta_{f}$ is trivial:

$$
\theta_{f}=\left(R_{0}\right)_{\theta_{f}}^{T}\left(q_{f}-q_{0}\right) .
$$

This solution can be used to perform the projection for $\delta_{f}$ :

$$
\delta_{f}=\left(\Psi^{q_{f}} \delta_{f}\right)^{-1}\left(\left(R_{0}\right)^{T}\left(q_{f}-q_{0}\right)-\rho_{f}(\theta)\right) .
$$

By applying a proper selection and normalization of $\delta_{f}$, also the matrix inversion in this equation can be eliminated. In order to allow an easy transformation, the projection modes $\Psi^{q q_{s e}, f}$ should be normalized in their respective interface DOFs and orthogonal to each other for their other interface DOFs.

For the above described transformation from super-element coordinates $q_{s e}$ to sub-system $q_{s s}$ coordinates, also a velocity transformation can be defined:

$$
\dot{q}_{s s}=\frac{\partial q_{s s}}{\partial q_{s e}} \dot{q}_{s e}=E\left(q_{0}, q_{f}, \theta_{i}\right) \dot{q}_{s e} .
$$

The projection matrix $E$ is a square matrix with the dimensions of the reduced model. This coordinate transformation is crucial for the correct derivation of the inertial forces, as described in Sec. 2.2.2.

An important point of attention is the definition of the interface modes with the use of a constant reduction space. In this case, the interface modes cannot be included in the total SVD-projection, as described in Sec. 2.1.1, because then their distinct meaning vanishes and no projection is possible any more. The method outlined in Sec. 2.1.1 is performed on the set of all internal modes and a separate SVD decomposition is performed for each interface mode and the single most dominant contribution is kept for each interface mode.

2.2.2. SE-GMP equations of motion. From the above described projections, the equations of motion for the super-element GMP coordinates $q_{s e}$ can be constructed. Again these equations can be obtained by applying Hamilton's principle to the Lagrangian of the system, as in Sec. 2.1.2.

: Inertial forces The inertial forces are defined based on the original unreduced mass-matrix with respect to the CRF. In order to construct the kinetic energy of the component, the reduced velocities have to be projected on the unreduced velocities expressed in the CRF $\dot{q}^{\prime}$ :

$$
\dot{q}^{\prime}=C\left(q_{s s}\right) \dot{q}_{s s},
$$

and a more elaborate description of the projection matrix $C\left(q_{s s}\right)$ is given by Naets [6]. For the super-element description however, an additional projection is required, which leads to:

$$
\dot{q}^{\prime}=C\left(q_{s s}\right) E\left(q_{0}, q_{f}, \theta_{i}\right) \dot{q}_{e s} .
$$

This equation can be inserted in the description of the kinetic energy in order to derive the inertial forces analogously as presented in Sec. 2.1.2. The description of the inertial forces include many configuration dependent projections. To limit the computational load, a set of mass invariants dependent on $\theta$ can be defined, as described in [6]. Through the use of these invariants the computational load becomes independent of the size of the unreduced system and can be considerably reduced. The reduced mass-matrix $M^{q_{s e} q_{s e}}$ can be computed as:

$$
M^{q_{s e} q_{s e}}=E^{T} D^{T}\left(M_{1}^{q_{s s} q_{s s}}+\sum_{i=1}^{n^{\delta}} M_{2 i}^{q_{s s} q_{s s}} \delta^{i}+\sum_{i=1}^{n^{\delta}} \sum_{j=1}^{n^{\delta}} M_{3 i j}^{q_{s s} q_{s s}} \delta^{i} \delta^{j}\right) D E .
$$


In this equation $D$ (see Eq. (33)) is a $n^{q_{s s}} \times n^{q_{s s}}$ projection matrix dependent on $p_{0}$ to take the orientation of the CRF into account. In order to speed up simulation and reduce the storage requirements with minimal loss of accuracy, the second-order terms in $\delta$ can be neglected. Inertial force on SE-GMP DOF $q_{s s}^{k}$ can then be evaluated as:

$$
g_{\text {iner }}^{q_{s e}}=M^{q_{s e} q_{s e}} \ddot{q}_{s e}
$$

$$
+\left(\sum_{l=1}^{n^{q_{s e}}} \frac{\partial M^{q_{s e} q_{s e}}}{\partial q_{s e}^{l}} \dot{q}_{s e}^{l}-\frac{1}{2} \frac{\partial\left(\dot{q}_{s e}\right)^{T} M^{q_{s e} q_{s e}}}{\partial q_{s e}}\right) \dot{q}_{s e} .
$$

Also these computations can be simplified considerably when a constant reduction space is used for the local GMP reduction.

: Internal forces The internal elastic forces $F_{i n t}^{q_{s e}}$ can be easily obtained by properly projecting the forces from Sec. 2.1.2. This leads to:

$$
g_{\text {int }}^{q_{s e}}=E^{T}\left[\begin{array}{c}
0_{6 \times 1} \\
g_{\text {int }, 0}^{\eta}+K^{\eta \eta} \eta
\end{array}\right] .
$$

In order to evaluate these forces, the SE-GMP coordinates first have to be transformed to the SSGMP coordinates to obtain the full $\eta$-vector.

: External forces The external loads $g_{\text {ext }}^{q_{s e}}$ on the reduced DOFs are also obtained by projection, but the exact form of this projection depends on the way the external forces are defined, e.g. with respect to what reference frame etc.

The resulting equations of motion are slightly more complicated than those described in SS-GMP coordinates due to the presence of the projection matrix $E$, but this added complexity is easily offset by the simple expressions for the constrained connection with other components.

2.3. Full SE-GMP modeling scheme. A complete SE-GMP reduced simulation can be split up into two main parts:

- A preprocessing in which the original model is reduced according to the GMP formalism, depicted in Fig. 4. In case the constant SVD based projection space is used, this can be calculated from the variable projection space and used for the projection of the system matrices.

- An actual simulation phase in which the SE-GMP component is evaluated by performing several coordinate transformations and evaluating the local GMP model by interpolation, as shown in Fig. 5.

This structure leads to a very efficient online-simulation approach, at the expense of a relatively computationally involved preprocessing phase from which the local GMP model is constructed. In the next section, this scheme is applied to a slider-crank mechanism with a highly flexible crank.

\section{NUMERICAL VALIDATION}

In order to demonstrate the use of the SE-GMP approach, a numerical validation is performed. The system under consideration is a planar slider-crank with a very slender crank and a very stiff connecting rod and heavy slider, as shown in Fig. 6. In this validation, the slender crank is the component which will exhibit strongly nonlinear behavior. Due to the large loads applied by the heavy connecting rod and slider, the crank will show large deformations such that nonlinear stiffening effects become apparent. In this case, only nonlinear flexible behavior is considered. Nonlinear material behavior, such as plasticity or 
Calculate deviation modes

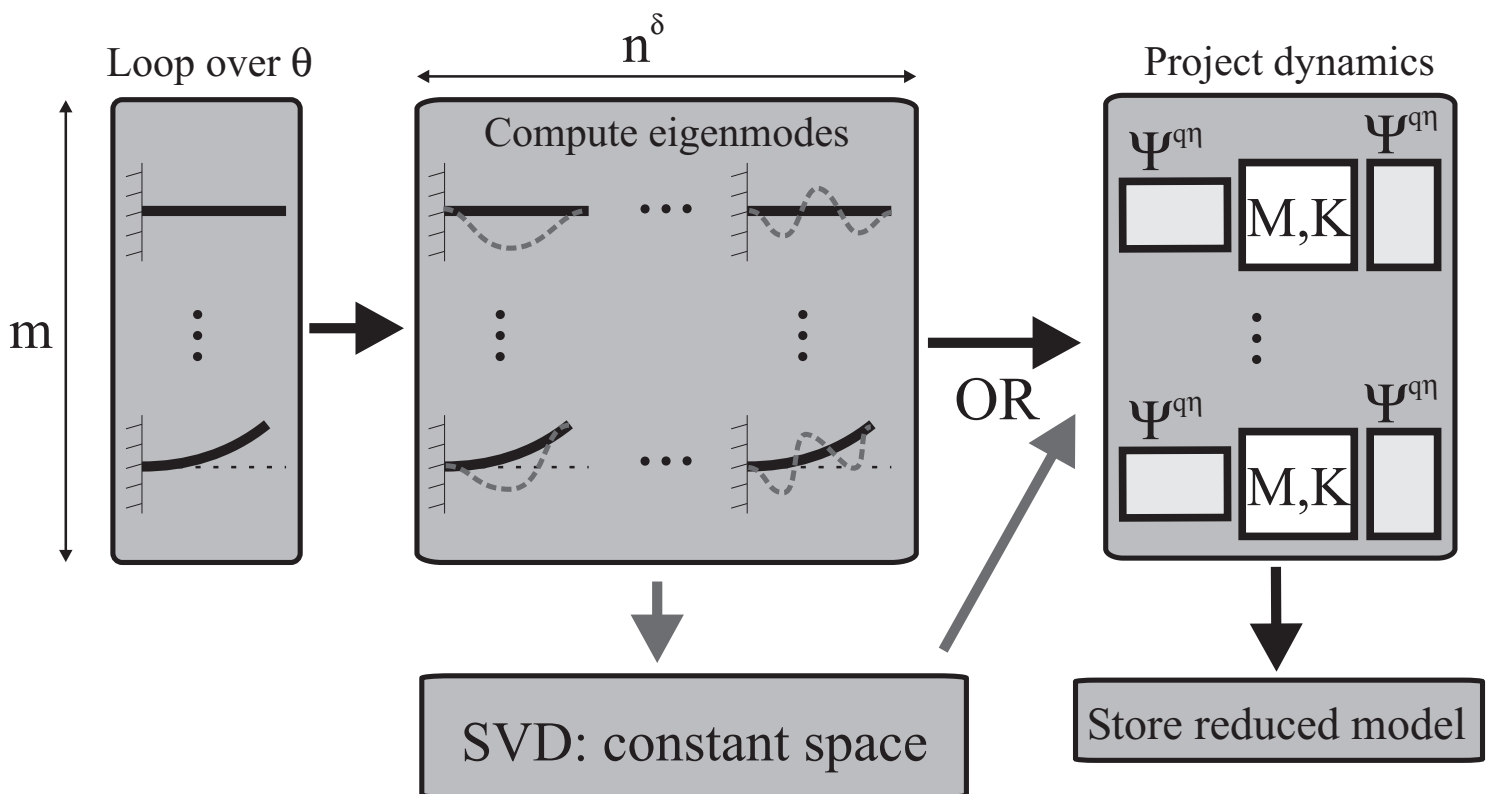

FIGURE 4. Flowchart of GMP reduction preprocessing

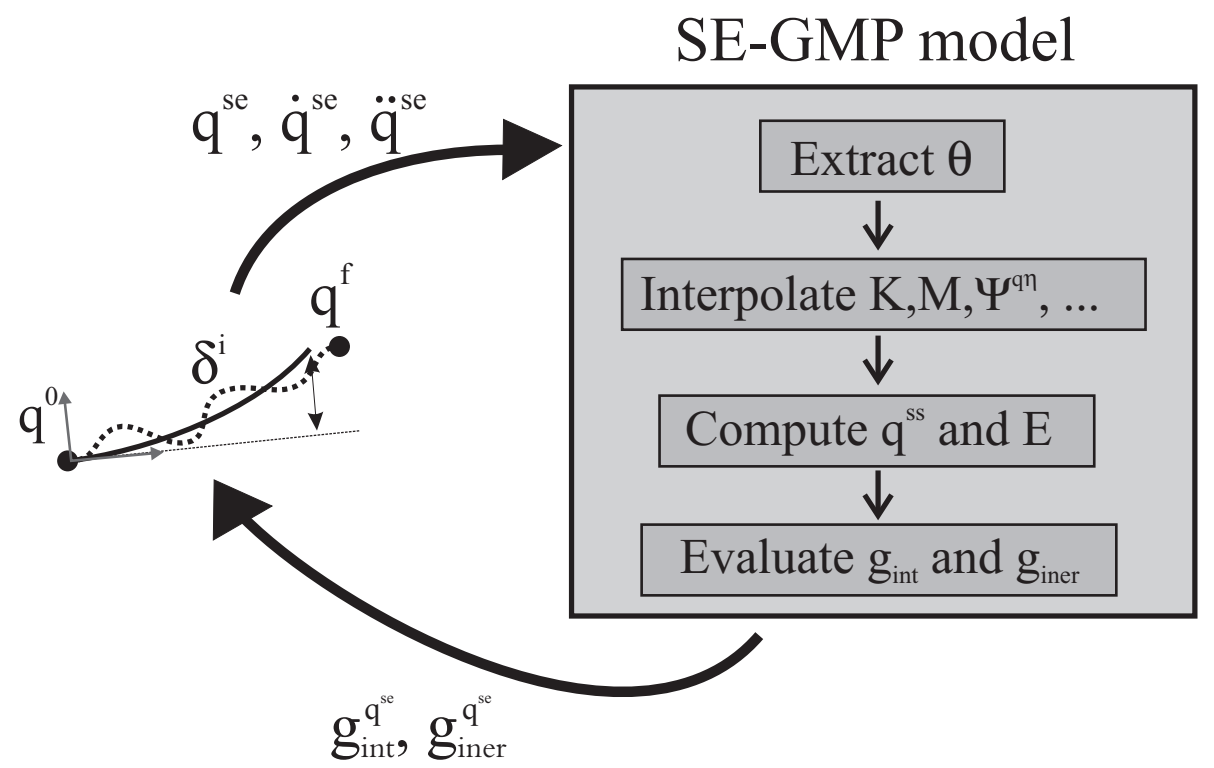

FIGURE 5. Flowchart of SE-GMP simulation

damage, is not included in the model. The proposed system is represented by a planar model and the system properties are depicted in Table 1. In this validation, the original model is constructed using a geometrically nonlinear beam model, as proposed by Géradin and Cardona [18] and a sufficiently high number of elements is required for the crank in order to capture the nonlinear deformation properly.

The unreduced nonlinear finite-element model serves as a reference for three different models in which the crank is reduced using different techniques:

- linear model reduced with Component Mode Synthesis (CMS),

- SE-GMP reduced model with variable projection space (SE-GMP-var), 


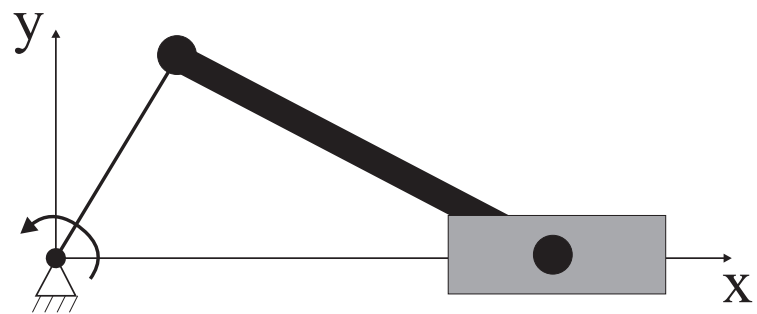

FIGURE 6. Slider-Crank mechanism for numerical validation.

TABLE 1. Parameters for slider-crank mechanism

\begin{tabular}{|c|ccc|}
\hline & Crank & Connecting Rod & Slider \\
\hline Length $[\mathrm{m}]$ & 0.4 & 0.6 & - \\
Area $\left[\mathrm{cm}^{2}\right]$ & 0.5 & 100 & - \\
Young's modulus $[\mathrm{Pa}]$ & $210 e 9$ & $210 e 9$ & - \\
Shear coefficient $[\mathrm{Pa}]$ & $80 e 9$ & $80 e 9$ & - \\
\# elements [/] & 30 & 4 & - \\
mass $[\mathrm{kg}]$ & 0.156 & 46.8 & 10 \\
\hline
\end{tabular}

- SE-GMP reduced model with constant projection space (SE-GMP-const).

The model with the linearly reduced crank is based on a floating-frame-of-reference (FFR) description of the crank with a modal description for the flexible behavior. A tangent frame approach is adopted where the reference frame is attached to the first node of the crank. For the modal reduction, no fixed-fixed eigenmodes of the system are considered and 3 interface modes are used for the connection between the crank and the connecting-rod. In this case a minimal number of modes provides the best accuracy because this stiffens the model, leading to a better approximation of the model with geometric stiffening. Since the original crank-model has 93 DOFs and the linearized crank model 6 DOFs (3 flexible +3 rigid), a considerable reduction is achieved. The aim of this validation is not to show the full potential of the speed-up by model reduction, but to focus on capturing the important dynamic effects in the reduced model.

For both SE-GMP models, the component-reference-frame is also attached to the first node of the crank. The dominant nonlinear motion of the system is expected to be the large bending motion of the beam. In order to parameterize this motion, the lateral deflection of the connection node with the connecting-rod with respect to the CRF is chosen as $\theta$. In this case this is also an interface DOF $\theta^{f}$. The $\theta$ is discretized with a discretization step of $\Delta \theta=0.001 \mathrm{~m}$ for the construction of the reduction space. For the modeling of the deviation with respect to this nonlinear motion, 3 additional linearized modes are used for the model with variable projection space. These consist of 1 dynamic internal mode and 2 additional interface modes (the CRF takes care of the interface with the ground and the lateral interface DOF is handled by $\theta^{f}$ ). For the variable reduction space, the dynamic modes are the fixed-fixed eigenmodes of the component. For the model with the constant reduction space, the dynamic modes are based on a singular value decomposition of the space spanned by these 3 modes of the component for all configurations. Based on the decay of the singular values, shown in Fig. 7, a mode-set with 15 constant modes is selected to approximate the variable space. The gradual decay of the singular values shows that it is difficult to get an accurate approximation of the variable projection space in this case and the model reduction is compromised by moving to a constant 


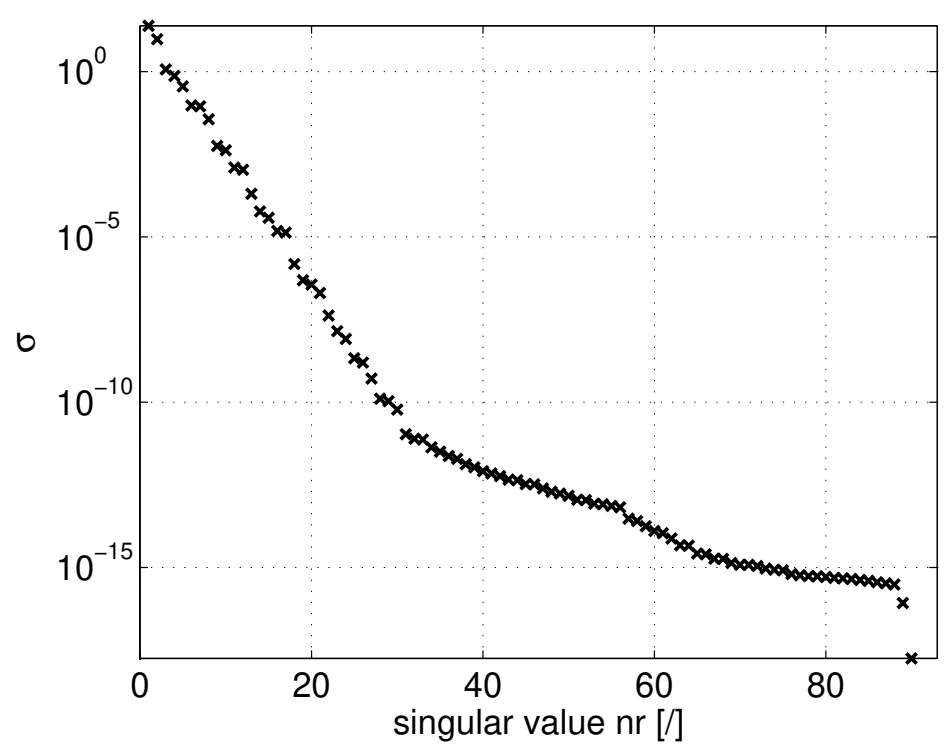

FIGURE 7. Singular values for dynamic projection space for crank.

projection space. For each interface DOF, one interface mode is used. This mode is the first mode from the SVD of the space spanned by all the static response modes over the different configurations for that DOF.

The presented validation of the proposed methodology for nonlinear component reduction is split into 2 cases:

- validation of the component behavior after GMP reduction,

- validation of the system behavior with the SE-GMP reduced component.

The first case is treated in Sec. 3.1. Here the crank is considered as a cantilever beam where the focus is on accurately describing the dynamics of the reduced component. The second case is presented in Sec 3.2, where the behavior of the full slider-crank mechanism is investigated.

All simulations are performed in MATLAB using a generalized $\alpha$-solver with a constant time-step [21].

3.1. GMP: Nonlinear cantilever beam. In this section, the effect of a GMP component reduction on the component responses are investigated. The component under investigation, is the flexible crank of the above described slider-crank mechanism. In order to investigate the dynamics of only the crank, this component is considered as a cantilever beam with a lateral force applied to its tip, as shown in Fig. 8. For the excitation

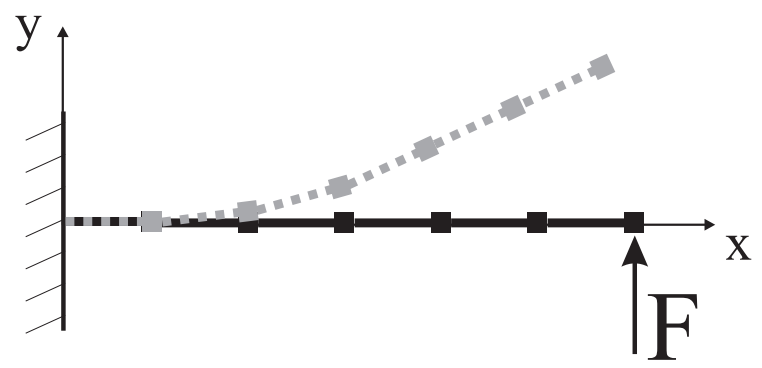

FIGURE 8. Crank as a cantilever beam to investigate its dynamics.

of this cantilever beam, two different tip loads $F$ are considered, as shown in Fig. 9. The first load (Fig. 9a) 


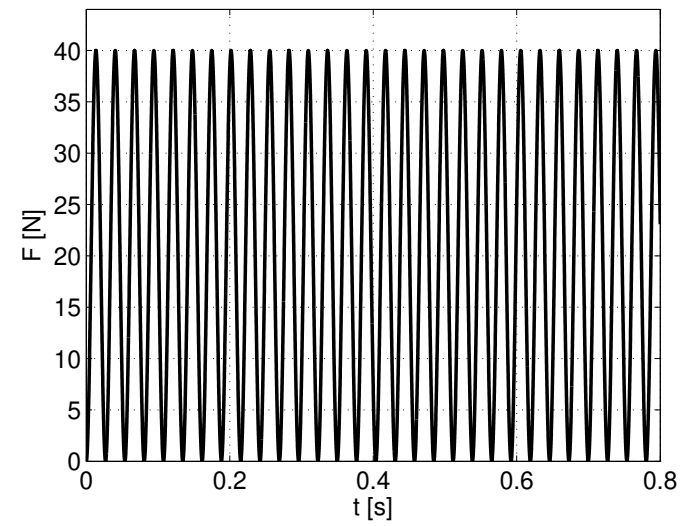

(A) Resonance load

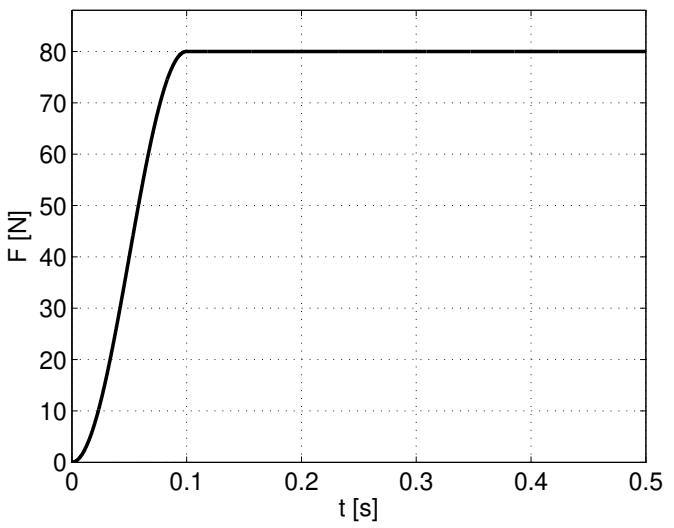

(B) Step load

FIGURE 9. Loads for cantilever beam

is aimed at exciting the first eigenfrequency of the cantilever. This simulation will show both the accuracy of the different simulation approaches and also highlight the stability properties. The second simulation (Fig. 9b) is a step response which will both provide some insights in the static response of the system and also show the behavior for free vibration. The simulations are performed with a generalized $\alpha$-solver with a constant step size $h=0.1 m s$ and $\rho_{\infty}=0.8$.

3.1.1. Simulation results. After both simulations, the tip-responses for both cases are compared for the four different models under consideration. These results are depicted in Fig. 10 and Fig. 11. For the resonance

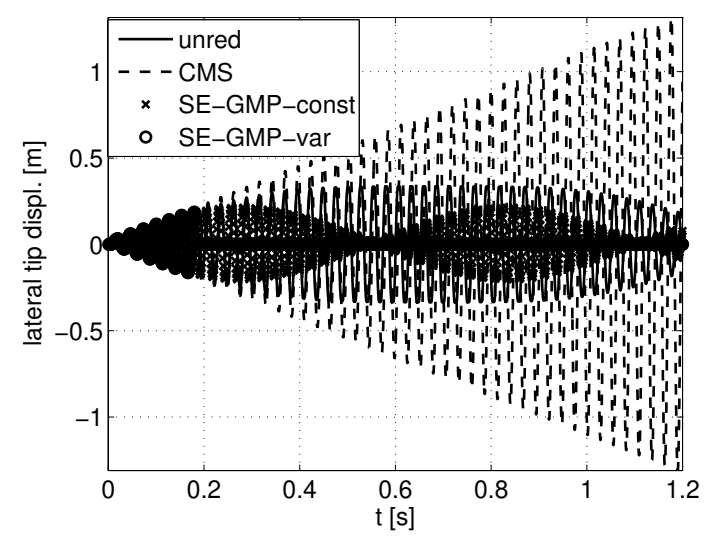

(A) Response resonance

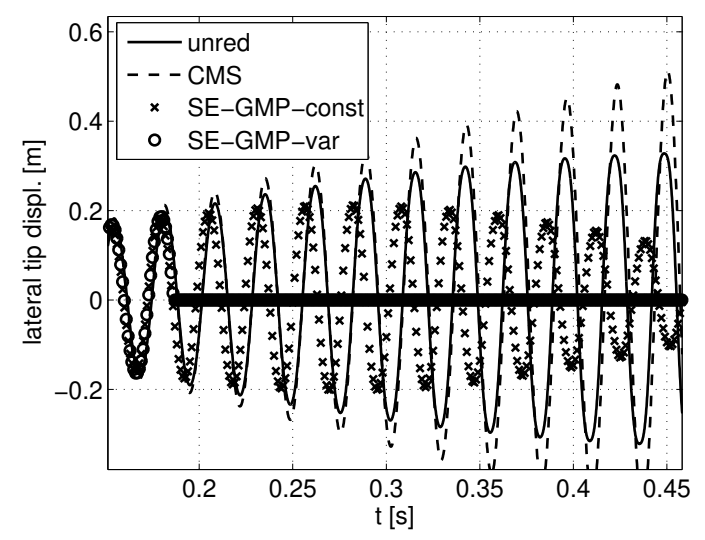

(B) Zoomed view

FIGURE 10. Tip response of cantilever beam under resonance.

load (Fig. 10a and Fig. 10b), it becomes apparent that the linear CMS reduced model is not capable of accurately capturing the nonlinear behavior of the unreduced component. In the case of a linear model under resonance conditions, the deflection amplitude will keep rising without a limit. This is clearly not a realistic behavior and so the linear model is not trustworthy in this context. This case also demonstrates the possible issues with the SE-GMP model with the variable reduction space. Initially the response of this model is accurate, but after a certain time $(0.18 s$, no convergence is reached after this time and the results 
are set to zero in the figure) the simulation crashes. At this time the velocity of $\theta$ becomes substantial and convergence problems arise due to numerical problems associated with the use of the derivatives of the projection space, as discussed in Sec. 2.1.2. The model with constant reduction space captures the global system behavior of the nonlinear model by showing a maximum deformation and then decreasing again. A frequency shift, as predicted in Sec. 2.1.1, is visible, but the general behavior of the unreduced original model is clearly captured in this model.

All models appear to deliver accurate results for the step load, as shown in Fig. 11a. In order to better judge the accuracy, a zoom on the transition from rising force to constant force is shown in Fig. 11b. This

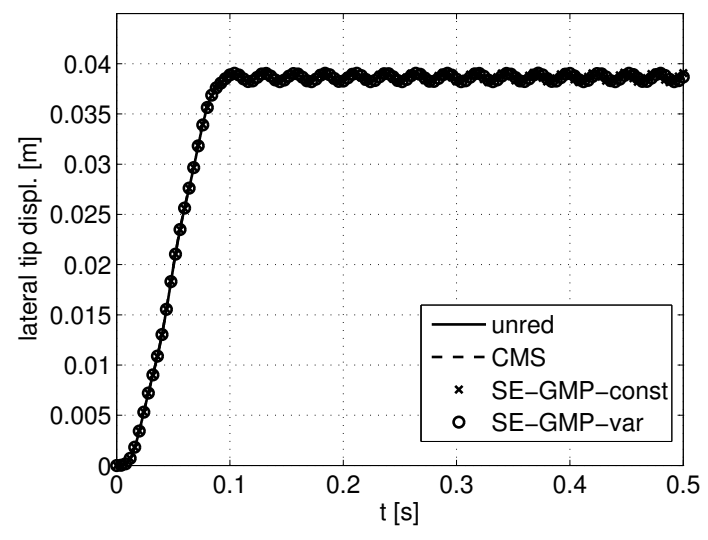

(A) Response step

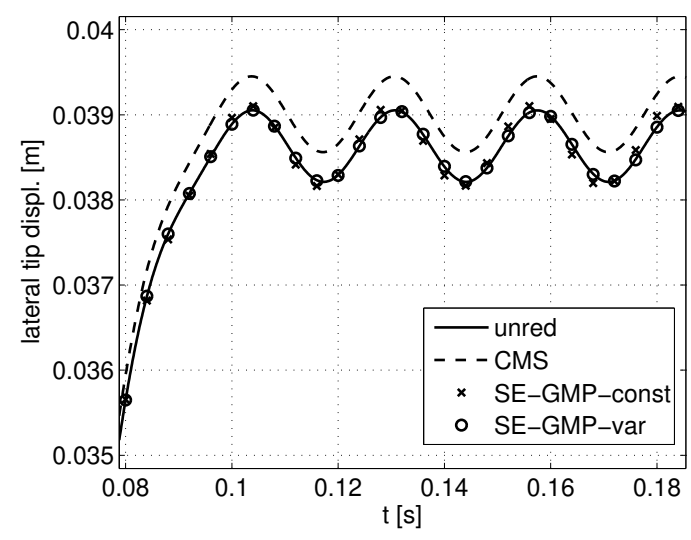

(B) Zoomed view

FIGURE 11. Tip response of cantilever beam under step-load.

zoomed view shows that again the linearly reduced CMS model is not capable of accurately describing the nonlinear behavior of the system due to lack of stiffening effects. In this case, both SE-GMP models provide accurate steady-state responses. Also here it is apparent that while the model with a variable reduction space provides an accurate approximation of the eigenfrequencies of the component, the constant reduction space leads to some stiffening of the linearized behavior, leading to a slight shift in the dynamic response.

These two validations on a geometrically stiffening cantilever beam show that the proposed SE-GMP reduction technique is capable of representing effects which cannot be represented by linearly reduced models. The SE-GMP model with a constant projection space is demonstrated to be more robust than the model with a variable one, but at the expense of a shifted dynamical behavior.

3.2. SE-GMP: Slider-crank mechanism. In this validation, the previously reduced component model is inserted in the simulation of the slider-crank mechanism (Fig. 6). In order to drive the mechanism, a torque, with the course depicted in Fig. 12, is applied to the first node of the crank. Due to the high relative flexibility of the crank and the high driving torque, this component exhibits large deformations. This case is chosen to examine the effect of the nonlinearity of one component on the global system response. The simulations are performed with a time step of $h=1 \mathrm{~ms}$ and spectral radius $\rho_{\infty}=0.8$.

3.2.1. Simulation results. Fig. 13 shows four configurations of the slider crank during the simulation for the four investigated modeling approaches. This figure clearly demonstrates that the model with the linearly flexible CMS component is not capable to describe the behavior of the proposed system. Due to the large deformations of the crank with the linear model the connecting rod overshoots and the system ends up in a 


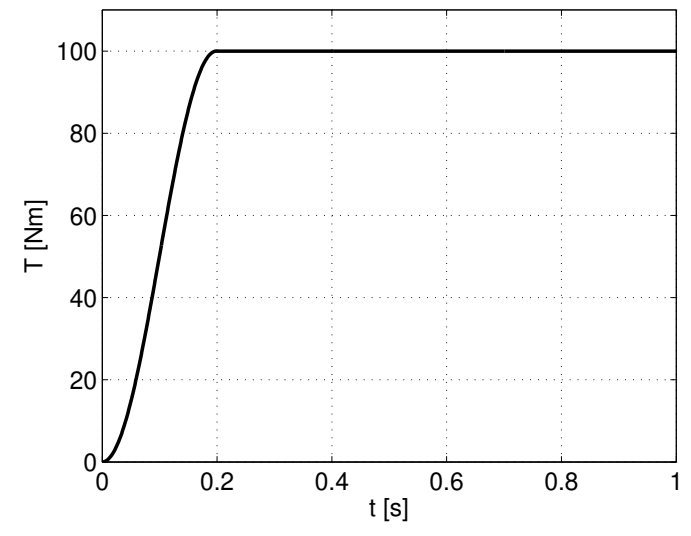

FIGURE 12. Driving torque on crank-slider mechanism.
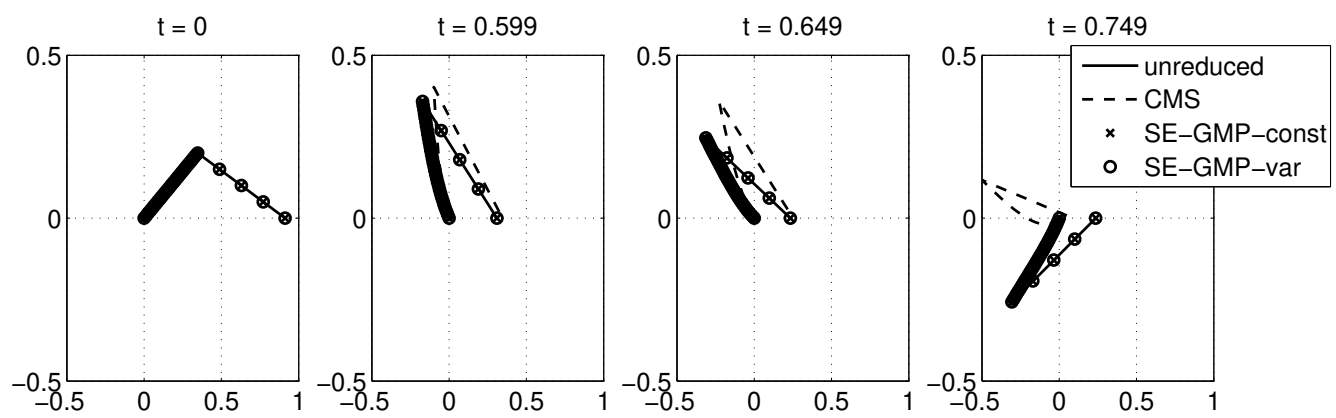

FIGURE 13. Snapshots of motion of the slider-crank mechanism.

different configuration. The unreduced nonlinear FE model and the two models with the SE-GMP reduced component show a good correspondence. The large deformation of the crank, which is clearly visible, is important, but due to the nonlinear geometric stiffening it remains considerably smaller than predicted by the linearized model.

The course of the crank angle, the slider position and their respective errors with respect to the unreduced model are depicted in Fig. 14. This figure again shows that the behavior of the model with the linearized component is unreliable and that both models with the SE-GMP reduced component provide reliable results both for the crank motion and for the full system behavior. The errors remain limited to 1 percent for the simulations with the SE-GMP component. Similar conclusions can also be drawn from the flexible deformation of the crank tip, as shown in Fig. 15. Both SE-GMP models provide accurate results, but the model with the constant projection space appears to provide slightly more accurate results. In this case, this is due to the more accurate description of the inertial effects for the SE-GMP model with the constant projection space. For this model, no numerical derivatives of the projection space are required, which eliminates an important source of error and this effect is visible in the presented results.

The slider-crank example clearly shows that the SE-GMP reduced component is capable of capturing nonlinear effect which cannot be contained in a linearly reduced model. Moreover, the slight advantage of the SE-GMP model with constant projection space, due to the more reliable inertial forces, is demonstrated through a better accuracy, but this comes at the cost of a larger model. 

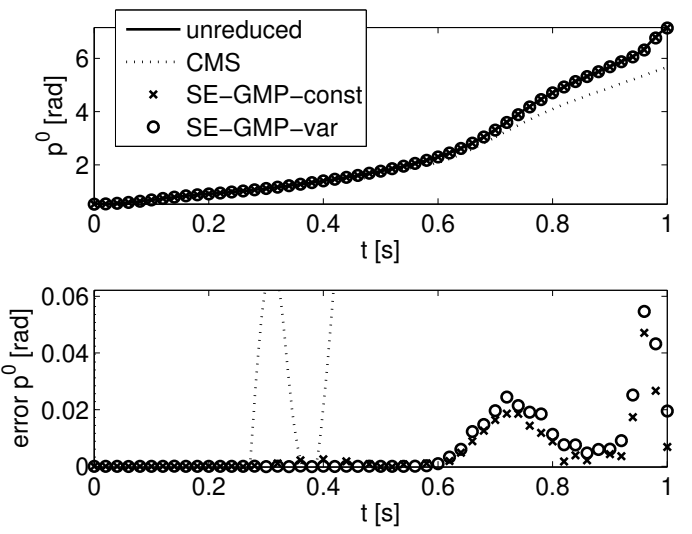

(A) Crank angle
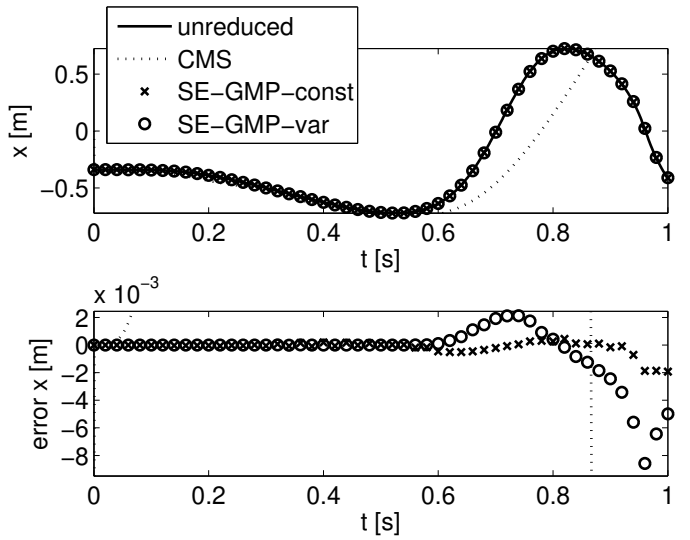

(B) Slider position

FIGURE 14. Response and error on response for slider-crank.
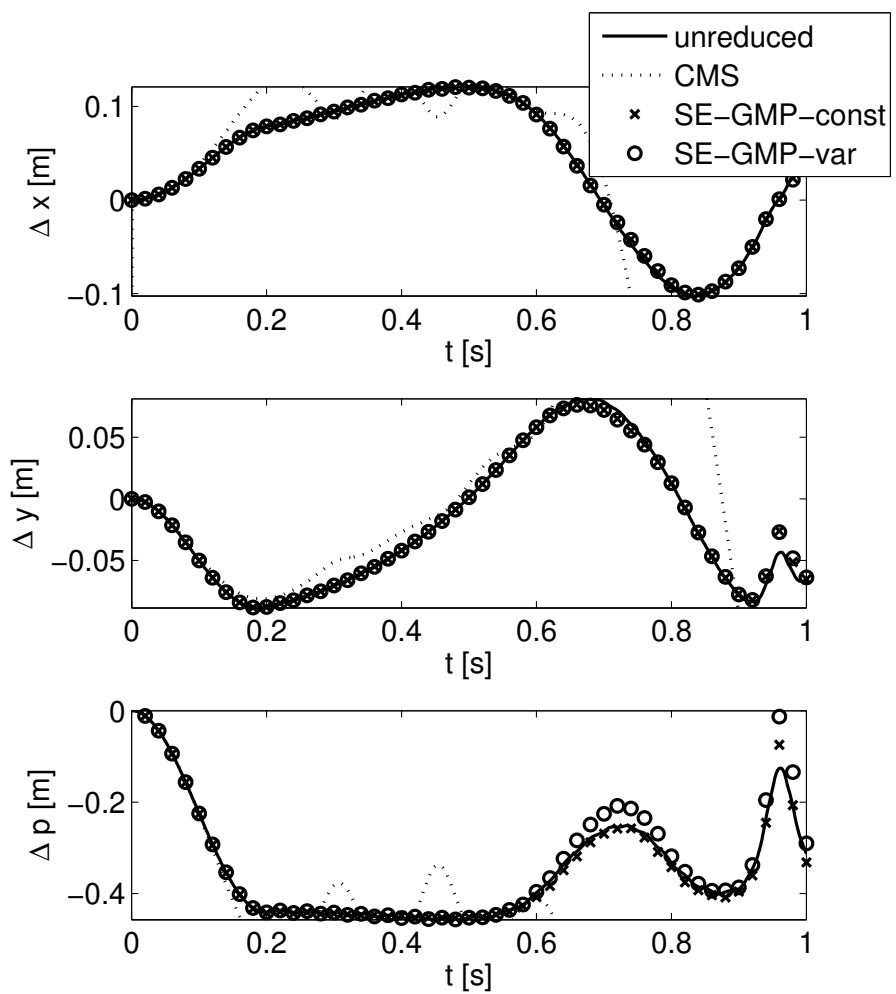

FIGURE 15. Flexible deformation of crank-tip.

\section{Conclusion}

In this work, a novel approach for the introduction of nonlinear components in multibody models is proposed. The Super-Element Global Modal Parameterization (SE-GMP) allows to construct nonlinear reduced models of components which can be efficiently introduced in and coupled with multibody models.

The model reduction is based on the GMP formalism, which is based on a nonlinear dynamic parameterization of a model. By describing the component model with respect to a component reference frame and 
by employing particular interface vectors for the projection space, the interface nodes of the reduced model can be expressed by their global coordinates. This last choice allows a very convenient interfacing with other multibody components. This work discusses the choice of projection space for this methodology, with a focus on the differences between a variable projection space and a constant projection space. Furthermore, the equations of motion, as derived from the Lagrangian of the system, are presented.

The proposed approach is validated on a planar slider crank mechanism with a strongly deforming crank. This example shows the inadequacy of linear reduction techniques, such as floating-frame-of-reference component mode synthesis, to describe this nonlinear component, while a SE-GMP model can accurately capture the behavior of this system.

Future research will focus on defining a generalized approach to add a wide range of nonlinearities (e.g. nonlinear damping) and investigations to optimized constant reduction bases. The current work has focused on geometric nonlinearities, but the framework should allow the reduction of material nonlinearities as well. In this case it will be necessary to generalize the definition of the nonlinear configuration parameter to other variables than positions.

\section{ACKNOWLEDGEMENTS}

The research of Frank Naets is funded by a Ph.D. grant of the Institute for the Promotion of Innovation through Science and Technology in Flanders (IWT-Vlaanderen).

\section{REFERENCES}

[1] T. Wasfy and A. Noor. Computational strategies for flexible multibody systems. Applied Mechanics Reviews, 56(6):553-613, 2002.

[2] J.A.C. Ambrosio and P. Verissimo. Improved bushing models for general multibody systems and vehicle dynamics. Multibody Syst. Dyn., 22(4):341-365, 2009.

[3] T.Z. Sinokrot, W.C. Prescott, M. Nembrini, and A. Toso. Multibody system modeling of flexible twist beam axles in car suspension systems. Proceedings of the ASME 2011 International Design Engineering Technical Conferences, Washington DC, USA, 29-31 August 2011.

[4] O.P. Agrawal and R. Kumar. A superelement model for analysis of multi-body system dynamics. Computers \& Structures, 32(5):1085-1091, 1989.

[5] O. Brüls, P. Duysinx, and J-C. Golinval. The global modal parameterization for nonlinear model-order reduction in flexible multibody dynamics. Int. J. Numer. Meth. Engng, 69(5):948-977, 2007.

[6] F. Naets, G.H.K. Heirman, and W. Desmet. Sub-system global modal parameterization for efficient simulation of flexible multibody systems. Int. J. Numer. Meth. Engng., 89(10):1227-1248, 2011.

[7] F. Naets, G.H.K. Heirman, and W. Desmet. A novel approach to real-time flexible multibody simulation: Sub-system global modal parameterization. Proceedings of the ASME 2011 International Design Engineering Technical Conferences, Washington DC, USA, 29-31 August 2011.

[8] F. Naets, T. Tamarozzi, and W. Desmet. Real-time flexible multibody simulation with global modal parameterization. Multibody Syst. Dyn., 27(3):267-284, 2012.

[9] F. Naets, G. H. K. Heirman, D. Vandepitte, and W. Desmet. Inertial force term approximations for the use of global modal parameterization for planar mechanisms. Int. J. Numer. Meth. Engng, 85:518-536, 2010.

[10] M. Lehner and P. Eberhard. A two-step approach for model reduction in flexible multibody dynamics. Multibody Syst Dyn, 17(2-3):157-176, 2007.

[11] J. Fehr and P. Eberhard. Simulation process of flexible multibody systems with non-modal model order reduction techniques. Multibody Syst. Dyn., 25(3):313-334, 2011.

[12] G.H.K. Heirman, F. Naets, and W. Desmet. How mode veering and mode crossing affects global modal parametrization and solutions to overcome these problems. 25-27 May 2010.

[13] Y.C. Liang, H.P. Lee, S.P. Lim, W.Z. Lin, K.H Lee, and C.G. Wu. Proper orthogonal decomposition and its applications - part 1: Theory. Journal of Sound and Vibration, 252(3):527-544, 2002. 
[14] S.E Boer, D. ten Hoopen, R.G.K.M Aarts, W.B.J. Hakvoort, and J.B. Jonker. Model reduction for efficient time-integration of planar non-linear flexible multibody models. Proceedings of the second joint international conference on Multibody System Dynamics, Stuttgart, Germany, May 29-June 12012.

[15] M.A. Neto, J.A.C. Ambrosio, and R.P. Leal. Sensitivity analysis of flexible multibody systems using composite materials components. Int. J. Numer. Meth. Engng., 77(3):386-413, 2009.

[16] D. Amsallem, J. Corial, K. Carlberg, and C. Farhat. A method for interpolating on manifolds structural dynamics reduced-order models. Int. J. Numer. Meth. Engng, 80(9):1241-1258, 2009.

[17] R. Eid, R. Castañé Selga, H. Panzer, T. Wolf, and B. Lohmann. Stability-preserving parametric model reduction by matrix interpolation. Mathemattical and Computer Modelling of Dynamical Systems, 17(4):319-335, 2011.

[18] M. Geradin and A. Cardona. Flexible Multibody Dynamics: A Finite Element Approach. J. Wiley and Sons, New York, 2001.

[19] S.E. Boer, R.G.K.M. Aarts, J.P. Meijaard, D.M. Brouwer, and J.B. Jonker. A two-node superelement description for modelling of flexible complex-shaped beam-like components. Proceedings of ECCOMAS MULTIBODY DYNAMICS, Brussels, Belgium, 4-7 July 2011.

[20] R. R. Craig and M. Bampton. Coupling of substructures in dynamic analysis. AIAA Journal, 6(7):1313-1321, 1968.

[21] O. Brüls and M. Arnold. The generalized-alpha scheme as a linear multistep integrator: Towards a general mechatronic simulator. ASME Journal of Computational and Nonlinear Dynamics, 3(4), 2008. 